\title{
Communication
}

\section{Theoretical study of the adsorption behavior of L-amino acids on the iron surface}

\author{
Anton Kasprzhitskii 1,2,*, Georgy Lazorenko ${ }^{1,2}$, Tatiana Nazdracheva ${ }^{3}$,Victor Yavna ${ }^{1}$ \\ 1 Rostov State Transport University, Narodnogo Opolcheniya Sq., Rostov-on-Don, 344038, Russia \\ 2 Mineralica Limited Liability Company, Skolkovo Innovation Center, 42 Bolshoy Boulevard, Moscow, \\ 121205, Russia \\ 3 Southern Federal University, 5 Zorge str., Rostov-on-Don, 344090, Russia \\ * Correspondence: akasprzhitsky@yandex.ru; Tel.: +7-928-757-9539
}

\begin{abstract}
This research evaluates the inhibitory effect of L-amino acids (AAs) with different side chain lengths on Fe surface implementing Monte Carlo (MC) simulation. A quantitative and qualitative description of the adsorption behavior of AAs on the iron surface has been carried out. Calculations have shown that the absolute values of the adsorption energy of L-amino acids increase with side chain prolongation; they are also determined by the presence of heteroatoms. AAs from nonpolar and basic groups have the best adsorption ability to the iron surface, which indicates their highest inhibitory efficiency according to the results of MC simulation.
\end{abstract}

Keywords: corrosion; amino acids; inhibitors; steel; iron; Monte Carlo simulation.

\section{Introduction}

The effectiveness of a particular inhibitor depends on many parameters, such as the composition of the corrosive medium, $\mathrm{pH}$ value, temperature, contact time, chemical nature of the inhibitor, type of surface, etc. [1-3]. Recently, there has been a boost in the number of inquiries where theoretical methods based on quantum mechanical and molecular simulation are applied to determine the nature of the molecular interaction of inhibitors with a metal surface and to assess their effectiveness based on these data. These methods allow determining not only the mechanism of inhibitor adsorption on the metal surface (by identifying nucleophilic attack and electrophilic attack atomic centers that exchange electrons with the metal), but also how corrosive ions penetrate through inhibitor films formed on the metal [4-8]. This gives the possibility of computational predictive evaluation of a wide class of compounds to identify molecular structures with evident inhibitory properties.

The aim of this work is to carry out a systematic comparative theoretical survey of the adsorption behavior of L-amino acids (AAs) on the iron surface to identify promising candidates for the role of effective iron corrosion inhibitors using the Monte Carlo simulation.

\section{Computational details}

AAs structures have been taken from the public repository for chemical structures and their bioactivities by the National Center for Biotechnology Information (NCBI) [9]. The Fe (100) surface was selected to model the adsorption behavior of amino acids. An $8 \times 8 \mathrm{Fe}(100)$ surface consisting of 8 atomic layers was created based on a pre-optimized bulk structure with a lattice constant of $2.82 \AA$ [10]. A vacuum layer of $30 \AA$ was applied in the cells to exclude the influence of atomic layers associated with imposed periodic boundary conditions.

The adsorption behavior of AAs on the iron surface in the gas phase was investigated by Monte Carlo simulations employing the Adsorption Locator module of Material Studio software [11]. The adsorption locator determines the configuration of the adsorbed L-amino acid based on the Metropolis algorithm [12]. The search for the global minimum is based on the metaheuristic algorithm simulating annealing with automated temperature control and the following parameters: 10 temperature cycles with 100,000 steps for each $[13,14]$. The COMPASS force field was employed to optimize the structure of L-amino acids in the search for the minimum energy [15]. The Ewald 
summation method [16] was applied to describe the electrostatic interaction with an accuracy of 10-5 $\mathrm{kcal} / \mathrm{mol}$. Van der Waals interaction energies were calculated implementing the Atom-based method with a cubic spline truncation and cut off distance of $18.5 \AA$.

\section{Results and discussion}

The investigation of the adsorption behavior of L-amino acids on the Fe (100) surface has been carried out by the Monte Carlo (MC) method in the gas phase. Evaluation of the corrosion inhibition efficiency AAs is based on the analysis of the adsorption energy (Eads) of equilibrium low-energy configurations of the structure. The influence of the side chain type on the equilibrium configuration of L-amino acids during adsorption on the iron surface was determined on the basis of $\mathbf{E}_{\boldsymbol{a d s}}$ using the following formula:

$$
\mathbf{E}_{\text {ads }}=\mathbf{E}_{T}-\left(\mathbf{E}_{A}+\mathbf{E}_{S}\right),
$$

where $\mathbf{E}_{a d s}$ is the energy of the substrate-adsorbate configuration, $\mathbf{E}_{T}$ is the total energy of the system "L-amino acid/Fe(100) surface", $\mathbf{E}_{\boldsymbol{A}}$ and $\mathbf{E}_{\boldsymbol{S}}$ are the energy of the inhibitor molecule and of the iron surface, respectively. The results of calculating $\mathbf{E}_{a d s}$ are submitted in the diagram in Fig. 1. All values of the adsorption energies are negative, which means that the process can proceed spontaneously. The highest negative adsorption energy indicates the system with the most stable coating. In accordance with the AAs classification by the side chain type, the calculation results manifested in Fig. 1 are divided into 4 groups: nonpolar, polar, acidic and basic [17]. In each group, an increment in the absolute value of the adsorption energy of an amino acid on the Fe (100) surface is observed, due to a side chain prolongation and the number of heteroatoms. Thus, an increase in the side chain length, which correlates with the values of the adsorption energy of L-amino acids, indicates a multiplication of the number of the adsorption centers for the formation of bonds with the metal surface.

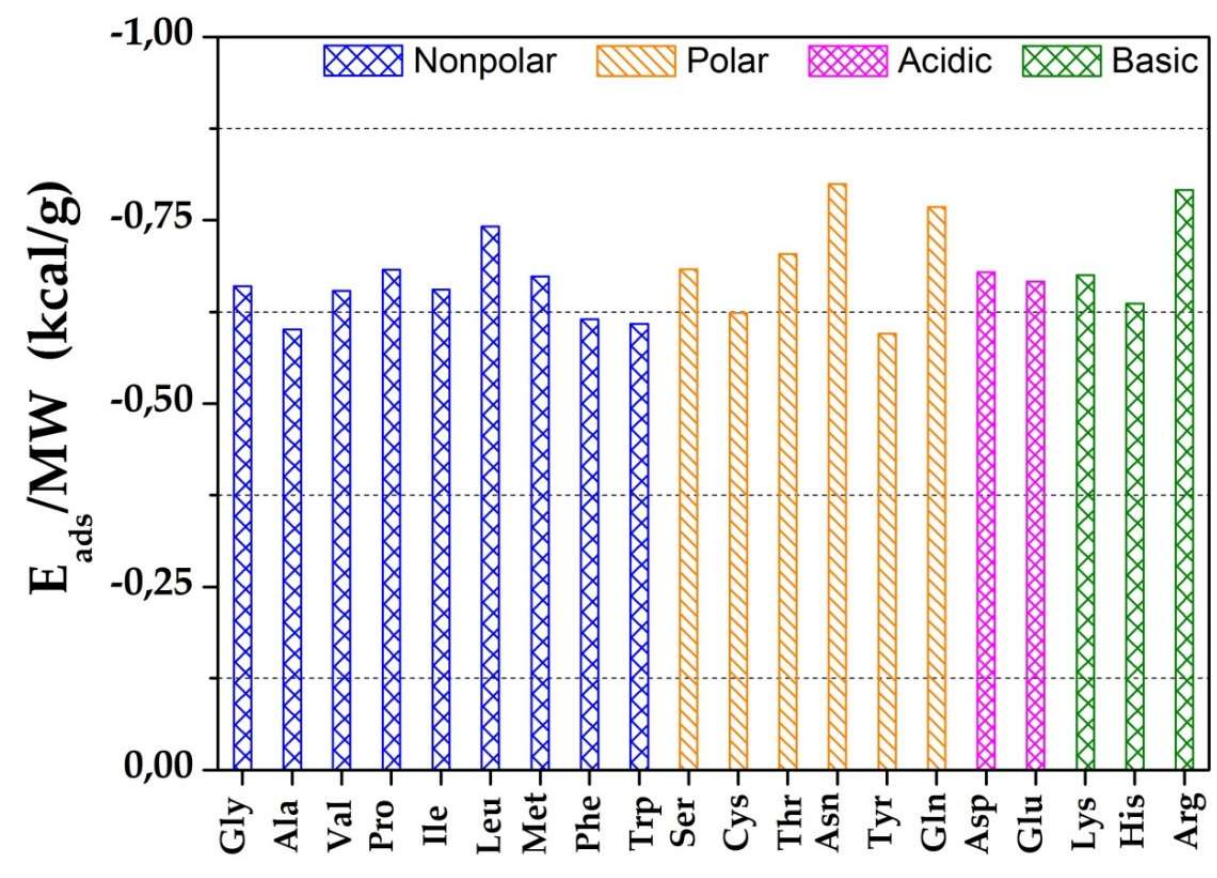

MW=Molecular Weight

Figure 1. The adsorption energy (Eads) obtained from Monte Carlo simulations of L-amino acids on Fe (100) surface in gas phase. 
The molecular structure of the side chain affects the nature of the formed bonds of L-amino acid with the metal surface and the affinity for it. The calculation demonstrated (Fig. 1) that the maximum absolute value of the adsorption energy of L-amino acids on the Fe (100) surface in accordance with the side chain type classification increases in the following sequence: Glu (acidic) < Gln (polar) < Trp (nonpolar) < Arg (basic). L-amino acids from nonpolar and basic groups have the best adsorption ability to the iron surface, which indicates their highest inhibitory efficiency according to the results of MC simulation.

\section{Conclusions}

In this work, we performed a comparative systematic analysis of the inhibitory effect of L-amino acids with different side chain lengths on the Fe (100) surface in the gas phase. Monte Carlo simulations have proved that AAs can be absorbed onto the iron surface, maximizing the number of bonds formed by being horizontally positioned. The absolute values of the energy of adsorption of these inhibitors on the Fe (100) surface increase with the side chain prolongation and the number of heteroatoms in it. The findings of theoretical studies have demonstrated the promising nature of using L-amino acids from nonpolar and basic groups as effective inhibitors of iron corrosion.

Author Contributions: Conceptualization, A.K. and G.L.; methodology, A.K.; software, A.K.; validation, A.K. and G.L.; investigation, A.K. and G.L.; writing-original draft preparation, A.K. and G.L.; writing-review and editing, A.K. and G.L.; visualization, T.N.; supervision, A.K.; project administration, V.Y. All authors have read and agreed to the published version of the manuscript.

Funding: The work was performed according to the state assignment of the Federal Agency for Railway Transport of the Russian Federation (protocol ROSZHELDOR of 15.04.2020 No.VCh-23).

Acknowledgments: The research is carried out using the equipment of the shared research facilities of HPC computing resources at Lomonosov Moscow State University.

Conflicts of Interest: The authors declare no conflict of interest.

\section{References}

1. Al-Amiery, A.A.; Kadhum, A.A.H.; Alobaidy, A.H.M.; Mohamad, A.B.; Hoon, P.S. Novel corrosion inhibitor for mild steel in HCL. Materials, 2014, 7 (2), 662-672. DOI: 10.3390/ma7020662

2. Odewunmi, N.A.; Mazumder, M.A.J.; Ali, S.A.; Aljeaban, N.A.; Alharbi, B.G.; Al-Saadi, A.A.; Obot, I.B. Impact of degree of hydrophilicity of pyridinium bromide derivatives on $\mathrm{HCl}$ pickling of X-60 mild steel: Experimental and theoretical evaluations. Coatings 2020, 10 (2), № 185, DOI: 10.3390/coatings10020185

3. Berisha, A.; Podvorica, F.I.; Mehmeti, V.; Syla, F.; Vataj, D. Theoretical and experimental studies of the corrosion behavior of some thiazole derivatives toward mild steel in sulfuric acid media. Maced. J. Chem. Chem. Eng. 2015, 34 (2), 287-294. DOI: 10.20450/mjcce.2015.576

4. Guo, L.; Qi, C.; Zheng, X.; Zhang, R.; Shen, X.; Kaya, S. Toward understanding the adsorption mechanism of large size organic corrosion inhibitors on an Fe(110) surface using the DFTB method. RSC Adv. 2017, 7 (46), 29042-29050. DOI: 10.1039/c7ra04120a

5. Hsissou, R.; Benhiba, F.; Abbout, S.; Dagdag, O.; Benkhaya, S.; Berisha, A.; Erramli, H.; Elharfi, A. Trifunctional epoxy polymer as corrosion inhibition material for carbon steel in $1.0 \mathrm{M} \mathrm{HCl}$ : MD simulations, DFT and complexation computations. Inorg. Chem. Comm. 2020, 115, № 107858. DOI: 10.1016/j.inoche.2020.107858

6. Hsissou, R.; Abbout, S.; Seghiri, R.; Rehioui, M.; Berisha, A.; Erramli, H; Assouag, M.; Elharfi, A. Evaluation of corrosion inhibition performance of phosphorus polymer for carbon steel in $[1 \mathrm{M}] \mathrm{HCl}$ : Computational studies (DFT, MC and MD simulations). J. Mater. Res. Technol. 2020, 9 (3), 2691-2703. DOI: 10.1016/j.jmrt.2020.01.002

7. Khaled, K.F. Monte Carlo simulations of corrosion inhibition of mild steel in $0.5 \mathrm{M}$ sulphuric acid by some green corrosion inhibitors. J. Solid State Electrochem. 2009, 13 (11), 1743-1756. DOI: 10.1007/s10008-009-0845-y 
8. Khaled, K.F.; Abdelshafi, N. S.; El-Maghraby, A. A.; Aouniti, A.; Al-Mobarak, N.; Hammouti, B. Alanine as Corrosion Inhibitor for Iron in Acid Medium: A Molecular Level Study. Int. J. Electrochem. Sci. 2012, 7, $12706-12719$.

9. Zerfaoui, M.; Oudda, H.; Hammouti, B.; Kertit, S.; Benkaddour, M. Inhibition of corrosion of iron in citric acid media by aminoacids. Prog. Org. Coat. 2004, 51 (2), pp. 134-138. DOI: 10.1016/j.porgcoat.2004.05.005

10. Khaled, K.F.; Abdel-Shafi, M.S. Chemical and electrochemical investigations of L-arginine as corrosion inhibitor for steel in hydrochloric acid solutions. Int. J. Electrochem. Sci. 2013, 8 (1), 1409-1421.

11. Perdew, J.P.; Burke, K.; Ernzerhof, M. Generalized gradient approximation made simple. Phys. Rev. Lett. 1996, 77 (18), 3865-3868. DOI: 10.1103/PhysRevLett.77.3865

12. Vanderbilt, D. Soft self-consistent pseudopotentials in a generalized eigenvalue formalism. Phys. Rev. B. 1990, 41 (11), 7892-7895. DOI: 10.1103/PhysRevB.41.7892

13. Wang, Y.; Wang, W.; Fan, K.-N.; Deng, J. Structural and electronic properties of silver surfaces: Ab initio pseudopotential density functional study. Surf. Sci. 2001, 490 (1-2), 125-132. DOI: 10.1016/S0039-6028(01)01320-6

14. Kruglikov, A.; Vasilchenko, A.; Kasprzhitskii, A.; Lazorenko, G. Atomic-level understanding of interface interactions in a halloysite nanotubes-PLA nanocomposite. RSC Adv. 2019, 9 (67), 39505-39514. DOI: $10.1039 /$ c9ra08772a

15. Garrity, K.F., Bennett, J.W., Rabe, K.M., Vanderbilt, D. Pseudopotentials for high-throughput DFT calculations. Comp. Mater. Sci. 2014, 81, 446-452. DOI: 10.1016/j.commatsci.2013.08.053

16. Pfrommer, B.G.; Côté, M.; Louie, S.G.; Cohen, M.L. Relaxation of Crystals with the Quasi-Newton Method. J. Comput. Phys. 1997, 131 (1), 233-240. DOI: 10.1006/jcph.1996.5612

17. Grimme, S. Semiempirical GGA-type density functional constructed with a long-range dispersion correction. J. Comp. Chem. 2006, 27 (15), 1787-1799. DOI: 10.1002/jcc.20495 